\title{
Bariatric Tele-screening During the COVID-19 Pandemic: Holding Back for Direct Approval?
}

\author{
Yentl Lodewijks $^{1} \mathbb{C} \cdot$ Simon Nienhuijs ${ }^{1}$
}

Received: 22 September 2021 / Revised: 25 November 2021 / Accepted: 13 December 2021 / Published online: 21 January 2022

(c) The Author(s), under exclusive licence to Springer Science+Business Media, LLC, part of Springer Nature 2021

\begin{abstract}
Purpose Many bariatric centers were restricted from providing routine care for outpatients. Telehealth visits allowed the continued care for outpatients and thus the preoperative screening for bariatric candidates. The objective of this study was to evaluate the effect of tele-screening on the multidisciplinary obesity team's decision (MDD) for bariatric surgery: disapproval, direct approval, or a recommendation for a prehabilitation program.

Materials and Methods Hospital data were collected from patients who underwent face-to-face or tele-screening for bariatric surgery between April and December 2020. The tele-screening cohort was then compared with a propensity-matched cohort of patients with face-to-face consultations. A chi-square and multinomial logistic regression analyses were performed.

Results After propensity matching, 396 patients remained for analysis. The majority received preoperative prehabilitation advice in both the tele-screening and face-to-face group (51\% versus 50\%). Although not significant, there were more direct approvals and fewer denials in the face-to-face group $(p=0.691)$. The multinomial logistic regression analysis showed no significant impact of tele-screening on the MDD result.

Conclusion Tele-screening in bariatric centers is feasible; the multidisciplinary team's decision was not significantly different between tele-screening and face-to-face screening which encourages the use of tele-screening in the future. An insignificant amount of fewer direct approvals and more denials were observed in the tele-screening group, which should be taken into account in future and larger case studies.
\end{abstract}

Keywords Telemedicine $\cdot$ Bariatric surgery screening $\cdot$ Multidisciplinary team

\section{Introduction}

The coronavirus disease 2019 (COVID-19) decreased healthcare utilization by a third [1]. The government briefly restricted bariatric centers to provide routine care for outpatients, and the World Health Organization (WHO) asked

\section{Key Points}

- Due to the COVID-19 pandemic, patients were tele-screened for bariatric surgery.

- Tele-screening and face-to-face consultations achieved similar MDD results.

- Although not significant, there was a trend for more disapproval by tele-screening.

Yentl Lodewijks

Yentl.lodewijks@catharinaziekenhuis.nl

1 Department of Obesity Surgery, Catharina Hospital Eindhoven, Michelangelolaan 2, 5623 EJ Eindhoven, The Netherlands to actively work on ways to protect people who are most at risk of severe disease [2]. As described in a systematic review by Huang et al., obesity increased the risk for hospitalization, intensive care unit admission, and death among COVID-19 patients [3]. The impact of COVID-19 on the bariatric tract was on the operative part and on the outpatient guidance pre- and postoperatively. To protect patients by reducing exposure to the hospital and social distancing, the role of telemedicine has increased substantially. Telehealth visits allow continued care for outpatients with obesity [4].

Although numerous articles tried to investigate the impact of COVID-19 and telehealth on bariatric surgery, they generally did not include its effect on the obesity team's decision [5-8]. Preoperative multidisciplinary evaluation is highly recommended in clinical practice guidelines, such as clinical nutrition and psychosocial-behavioral evaluation [9-11]. In addition, some centers provide an additional prehabilitation program. 
Therefore, the objective of this study is to evaluate the effect of tele-screening on the multidisciplinary obesity team's decision for bariatric surgery: a disapproval, direct approval, or a recommendation for a prehabilitation program

\section{Materials and Methods}

Hospital data were anonymously collected from patients who were screened for bariatric surgery between April and December 2020, during this period tele-screening was introduced using video consultations. According to the hospital board's policy, teleconsulting should be offered first, still leaving face-to-face contact as a possibility. The obesity team decided which patients received these physical consultations, for example, patients with no access to telehealth. After 3 months, these COVID regulations were loosened for 2 months. But due to rising COVID cases, teleconsulting took the reins again. As a result, one group underwent face-to-face consultations; the other group underwent telescreening. This multidisciplinary screening is performed by an obesity nurse, psychologist, dietician, and physiotherapist. In case of tele-screening, patients received three different video consultations and a separate telephone consultation from the physiotherapist. After both types of screening, these patients were multidisciplinary discussed (MDD) by the obesity team according to the IFSO guidelines to determine if a patient was approved for surgery, denied, or required an additional prehabilitation program by a dietitian and/or psychologist [12]. Patients with direct approval received a preoperative face-to-face consult with a surgeon, who informs on risks and complications and obtains informed consent. If a preoperative prehabilitation program was recommended, patients could refuse this program and return to their general practitioner. If the patient accepted, the program endured for 3-6 months before a bariatric procedure was scheduled. If the multidisciplinary team denied a patient for surgery, they were also referred back to primary care for conservative treatment.

Informed consent was obtained from all patients, which were recorded on the center's eHealth platform. Data on weight, height, age, quality of life, the MDD result, intoxications, and 19 comorbidities of which 6 obesity-related were collected. In the case of face-to-face consultations, height and weight were measured by a nurse instead of selfrapportage. Quality of life is measured with the OBESI-Q questionnaire, derived from the BODY-Q, consisting of 5 subscales on eating behavior, social functioning, psychological functioning, physical activities, and body image [13]. These scales were converted to a total score ranging from 0 to 100 , higher scores indicating better quality of life.

\section{Statistical Analysis}

Both the tele-screening and face-to-face consultation cohorts were analyzed for baseline characteristics. The tele-screening cohort was then compared with a propensity-matched cohort of patients with face-to-face consultations. Co-variables included were age, body mass index (BMI), gender, and comorbidities. Propensity scores were matched on a one-to-one basis with a caliper width of 0.1 to compare the multidisciplinary outcome among bariatric surgery candidates. Additionally, a chi-square and multinomial logistic regression analysis were performed to analyze if the type of screening was associated with the dependent variable, the MDD result; accepted for surgery, a preoperative prehabilitation program, or denied for surgery.

In normally distributed data, differences between telescreening and face-to-face consultations were tested using Pearson's chi-square test for categorical data and the one-way analysis of variance for continuous variables. Continuous variables in normally distributed data were presented as mean and standard deviation; not normally distributed data were reported as median and interquartile range.

Statistical analyses were performed using SPSS software (version 26). Significance levels were set for $p$ value $<0.05$.

\section{Results}

The results of 511 patients were extracted from the hospital database, after matching 396 patients remained for the analysis. The majority were female $304(76.8 \%)$ with a mean age of 42.1 years and a median BMI of $41.6 \mathrm{~kg} / \mathrm{m}^{2}$ (interquartile range (IQR) 39.0-45.2). The patient characteristics of both groups before and after matching are described in Table 1 . There were no significant differences between groups after matching.

Table 2 represents the MDD result; the majority received a preoperative prehabilitation program in both the tele-screening and face-to-face group (51\% versus 50\%). Although not significant, there were more direct approvals and fewer denials in the face-to-face group $(p=0.691)$. The multinomial logistic regression analysis in Table 3 also showed no significant impact of tele-screening on the MDD result. Compared to direct approval, the tele-screening group had an odds ratio (OR) of 1.383 (confidence interval (CI) [0.645-2.966], $p=0.404$ ) for being denied for surgery and an OR of 1.098 (CI [0.726-1.659], $p=0.658$ ) for receiving a prehabilitation program advice.

\section{Discussion}

Telemedicine has been promoted since 1995 when the modern telemedicine era arrived [14]. Telehealth was growing in popularity even before the COVID pandemic started, 
Table 1 Patient characteristics before and after propensity score matching (PSM). SD standard deviation, IQR interquartile range, GERD gastroesophageal reflux disease, $O S A S$ obstructive sleep apnea syndrome, $B M I$ body mass index

\begin{tabular}{|c|c|c|c|c|c|c|}
\hline & \multicolumn{3}{|c|}{ Before PSM $(n=511)$} & \multicolumn{3}{|c|}{ After PSM $(n=396)$} \\
\hline & Tele-screening & Face-to-face & $p$ value & Tele-screening & Face-to-Face & $p$ value \\
\hline Number of patients & $198(38.7 \%)$ & $313(61.3 \%)$ & & 198 & 198 & \\
\hline \multicolumn{7}{|l|}{ Demography } \\
\hline Gender & & & & & & 0.475 \\
\hline Male & $43(21.7 \%)$ & $84(26.8 \%)$ & 0.192 & $43(21.7 \%)$ & $49(24.7 \%)$ & \\
\hline Female & $155(78.3 \%)$ & $229(73.2 \%)$ & & $155(78.3 \%)$ & $149(75.3 \%)$ & \\
\hline Age, mean, years $(S D)$ & $41.8(11.9)$ & $42.5(11.9)$ & 0.825 & $41.8(11.9)$ & $42.4(11.9)$ & 0.508 \\
\hline$B M I$, median, $\mathrm{kg} / \mathrm{m}^{2}(I Q R)$ & $41.4(38.9-44.5)$ & $41.5(38.9-45.9)$ & 0.512 & $41.4(38.9-44.5)$ & $41.9(39.0-45.9)$ & 0.360 \\
\hline Hypertension & $9(4.5 \%)$ & $28(8.9 \%)$ & 0.062 & $9(4.5 \%)$ & $17(8.6 \%)$ & 0.105 \\
\hline Dyslipidemia & $5(2.5 \%)$ & $11(3.5 \%)$ & 0.532 & $5(2.5 \%)$ & $6(3.0 \%)$ & 0.760 \\
\hline Diabetes mellitus II & $35(17.7 \%)$ & $83(26.5 \%)$ & 0.021 & $35(17.7 \%)$ & $51(25.8 \%)$ & 0.051 \\
\hline GERD & $34(17.2 \%)$ & $55(17.6 \%)$ & 0.907 & $34(17.2 \%)$ & $36(18.2 \%)$ & 0.792 \\
\hline OSAS & $33(16.7 \%)$ & $55(17.6 \%)$ & 0.792 & $33(16.7 \%)$ & $36(18.2 \%)$ & 0.691 \\
\hline Musculoskeletal pain & $76(38.4 \%)$ & $117(37.4 \%)$ & 0.820 & $76(38.4 \%)$ & $78(39.4 \%)$ & 0.837 \\
\hline \multicolumn{7}{|l|}{ Intoxications } \\
\hline Drugs & $1(0.5 \%)$ & $6(1.9 \%)$ & 0.181 & $1(0.5 \%)$ & $3(1.5 \%)$ & 0.315 \\
\hline Alcohol & $140(70.7 \%)$ & $190(60.7 \%)$ & 0.021 & $134(67.7 \%)$ & $124(62.6 \%)$ & 0.292 \\
\hline Smoking & $37(18.7 \%)$ & $65(20.8 \%)$ & 0.567 & $37(18.7 \%)$ & $35(17.7 \%)$ & 0.794 \\
\hline Quality of life, mean, $S D$ & $35.85(11.6)$ & $36.21(9.4)$ & 0.030 & $36.52(10.5)$ & $36.28(9.7)$ & 0.368 \\
\hline
\end{tabular}

Table 2 The multidisciplinary discussion result for tele-screening and face-to-face consultations

\begin{tabular}{llll}
\hline & Tele-screening & Face-to-face & $p$ value \\
\hline Direct approval & $79(39.9 \%)$ & $85(42.9 \%)$ & 0.691 \\
Preoperative trajectory & $101(51 \%)$ & $99(50 \%)$ & \\
Denial at the time & $18(9.1 \%)$ & $14(7.1 \%)$ & \\
\hline
\end{tabular}

Table 3 Multinomial logistic regression analysis for the multidisciplinary discussion result and type of screening. $C I$ confidence intervals

\begin{tabular}{|c|c|c|c|c|}
\hline & \multicolumn{3}{|c|}{$95 \%$ CI for Odds Ratio } & \multirow[t]{2}{*}{$p$ value } \\
\hline & Lower bound & Odds Ratio & Upper bound & \\
\hline $\begin{array}{l}\text { Preoperative } \\
\text { counseling vs } \\
\text { approved for } \\
\text { surgery }\end{array}$ & & & & \\
\hline $\begin{array}{l}\text { Tele-screening } \\
\text { Denied for } \\
\text { surgery vs } \\
\text { approved for } \\
\text { surgery }\end{array}$ & 0.726 & 1.098 & 1.659 & 0.658 \\
\hline Tele-screening & 0.645 & 1.383 & 2.966 & 0.404 \\
\hline
\end{tabular}

but was still in an early age of development, particularly in low-income countries [15]. For this reason, the impact on the multidisciplinary outcome was still unknown. Because of the pandemic, the hospital was forced to provide telehealth, among them tele-screening for bariatric surgery. Consequently, the results of the multidisciplinary outcome could be compared in tele-screening versus face-to-face consultations. After propensity matching, there were no significant differences in the MDD result between telescreening and face-to-face screening, meaning healthcare providers did not come to a different judgment on bariatric surgery candidates. As the patient satisfaction for telehealth overall is relatively high, tele-screening can be the future for bariatric centers as it also decreases the burden on resources [16, 17].

The relationship between the type of screening and the MDD result was not significant; there were slightly more denials and fewer approvals in the group who were telescreened. One of the explanations could be that the use of tele-screening was new for both patient and healthcare provider and therefore odd to use. Additionally, sensory information and affective properties are compromised by the use of telemedicine technologies [18]. However, the sample size was relatively small and not randomized, therefore implicating caution in the interpretation of this result. Other limitations of this study are the capricious policy of 
loosening the COVID-19 regulations and the exceptions for face-to-face consultations in case of language barriers or no access to telehealth which results in selection bias. In our national guidelines, it was recommended not to invite patients with language barriers for video consultations in general. To overcome these limitations, a larger trial with unselected cases would help. The skills necessary for tele-screening will likely improve over time. With this improvement, the effectiveness of tele-screening could level or surpass face-to-face consultations. The results of the present study originated from a preliminary phase of tele-screening but were encouraging to continue to offer this modality and provided useful information for improvements in the future. After the first COVID waves, the obesity team preferred face-to-face consultations for logistic reasons and non-verbal communication. After discussing personal experiences and analyzing the data, tele-screening was concluded to be a good alternative in times of social distancing and is currently implemented in our practice. Patients can now choose between video consultations or face-to-face consultations as their screening procedure, and video consultations are now common practice for several postoperative follow-ups.

\section{Conclusion}

Tele-screening in bariatric centers is feasible; the multidisciplinary team's decision was not significantly different between tele-screening and face-to-face screening which is encouraging the use of tele-screening in the future. An insignificant amount of less direct approvals and more denials were observed in the tele-screening group, which should be taken into account in future and larger case studies.

\section{Declarations}

Ethics Approval All procedures performed in studies involving human participants were in accordance with the ethical standards of the institutional and/or national research committee and with the 1964 Helsinki Declaration and its later amendments or comparable ethical standards. The study is conducted in accordance to the standards of Good Clinical Practice, with Dutch law in general and with the Act Medical Research Involving Human Subjects in particular.

Consent to Participate Informed consent was obtained from all individual participants included in the study.

Conflict of Interest The authors declare no competing interests.

\section{References}

1. Moynihan R, Sanders S, Michaleff ZA et al. Impact of COVID19 pandemic on utilisation of healthcare services: a systematic review. BMJ Open. 2021;11(3):e045343. https://doi.org/10.1136/ bmjopen-2020-045343.

2. Chauhan V, Galwankar S, Arquilla B et al. Novel coronavirus (COVID-19): leveraging telemedicine to optimize care while minimizing exposures and viral transmission. J Emerg Trauma Shock. 2020;13(1):20-4. https://doi.org/10.4103/JETS.JETS_32_ 20.

3. Ho JSY, Fernando DI, Chan MY et al. Obesity in COVID-19: a systematic review and meta-analysis. Ann Acad Med Singap. 2020;49(12):996-1008. https://doi.org/10.47102/annals-acadm edsg.2020299.

4. Mehta P, Stahl MG, Germone MM et al. Telehealth and nutrition support during the COVID-19 pandemic. J Acad Nutr Diet. 2020;120(12):1953-7. https://doi.org/10.1016/j.jand.2020.07.013.

5. Bianciardi E, Imperatori $C$, Niolu $C$ et al. Bariatric surgery closure during COVID-19 lockdown in Italy: the perspective of waiting list candidates. Front Public Health. 2020;8:582699. https:// doi.org/10.3389/fpubh.2020.582699.

6. Lazaridis II, Kraljevic M, Schneider R et al. The impact of the COVID-19 pandemic on bariatric surgery: results from a worldwide survey. Obes Surg. 2020;30(11):4428-36. https://doi.org/10. 1007/s11695-020-04830-8.

7. Chao GF, Ehlers AP, Telem DA. Improving obesity treatment through telemedicine: increasing access to bariatric surgery. Surg Obes Relat Dis. 2021;17(1):9-11. https://doi.org/10.1016/j.soard. 2020.09.012.

8. Brown AM, Ardila-Gatas J, Yuan V et al. The impact of telemedicine adoption on a multidisciplinary bariatric surgery practice during the COVID-19 pandemic. Ann Surg. 2020;272(6):e30610. https://doi.org/10.1097/SLA.0000000000004391.

9. Mechanick JI, Apovian C, Brethauer S et al. Clinical practice guidelines for the perioperative nutrition, metabolic, and nonsurgical support of patients undergoing bariatric procedures - 2019 update: cosponsored by American Association of Clinical Endocrinologists/American College of Endocrinology, the Obesity Society, American Society for Metabolic \& Bariatric Surgery, Obesity Medicine Association, and American Society of Anesthesiologists - executive summary. Endocr Pract. 2019;25(12):134659. https://doi.org/10.4158/GL-2019-0406.

10. Thorell A, MacCormick AD, Awad S et al. Guidelines for perioperative care in bariatric surgery: Enhanced Recovery After Surgery (ERAS) Society recommendations. World J Surg. 2016;40(9):2065-83. https://doi.org/10.1007/s00268-016-3492-3.

11. Marshall S, Mackay H, Matthews $\mathrm{C}$ et al. Does intensive multidisciplinary intervention for adults who elect bariatric surgery improve post-operative weight loss, co-morbidities, and quality of life? A systematic review and meta-analysis. Obes Rev. 2020;21(7):e13012. https://doi.org/10.1111/obr.13012.

12. Fried M, Yumuk V, Oppert JM et al. Interdisciplinary European guidelines on metabolic and bariatric surgery. Obes Surg. 2014;24(1):42-55. https://doi.org/10.1007/s11695-013-1079-8.

13. Klassen AF, Cano SJ, Alderman A, Soldin M, Thoma A, Robson S, Kaur M, Papas A, Van Laeken N, Taylor VH, Pusic AL. The BODY-Q: a patient-reported outcome instrument for weight loss and body contouring treatments. Plast Reconstr Surg Glob Open. 2016;4(4):e679. https://doi.org/10.1097/GOX.0000000000 000665.PMID:27200241;PMCID:PMC4859238.

14. Weinstein RS, Krupinski EA, Doarn CR. Clinical examination component of telemedicine, telehealth, mHealth, and connected 
health medical practices. Med Clin North Am. 2018;102(3):53344. https://doi.org/10.1016/j.mcna.2018.01.002.

15. Marcolino MS, Oliveira JAQ, D'Agostino $M$ et al. The impact of mHealth interventions: systematic review of systematic reviews. JMIR Mhealth Uhealth. 2018;6(1):e23. https://doi.org/10.2196/ mhealth.8873.

16. Ramaswamy A, Yu M, Drangsholt S et al. Patient satisfaction with telemedicine during the COVID-19 pandemic: retrospective cohort study. J Med Internet Res. 2020;22(9):e20786. https://doi. org/10.2196/20786.
17. Tenforde AS, Borgstrom H, Polich G et al. Outpatient physical, occupational, and speech therapy synchronous telemedicine: a survey study of patient satisfaction with virtual visits during the COVID-19 pandemic. Am J Phys Med Rehabil. 2020;99(11):97781. https://doi.org/10.1097/PHM.0000000000001571.

18. Lupton D, Maslen S. Telemedicine and the senses: a review. Sociol Health Illn. 2017;39(8):1557-71. https://doi.org/10.1111/ 1467-9566.12617.

Publisher's Note Springer Nature remains neutral with regard to jurisdictional claims in published maps and institutional affiliations. 